\title{
Control and calibration recipes for photonic integrated circuits
}

\author{
Maziyar Milanizadeh, Sara Ahmadi, Matteo Petrini, Douglas Aguiar, Riccardo Mazzanti, Francesco \\ Zanetto, Emanuele Guglielmi, Marco Sampietro, Francesco Morichetti, and Andrea Melloni, Fellow, \\ OSA
}

\begin{abstract}
This paper presents the key ingredients and the best practices for implementing simple, effective and robust control and calibration procedures for arbitrary photonic integrated circuit (PIC) architectures. Three main features are presented and discussed: a technique to cancel out the effects of mutual crosstalk among thermal tuners, the exploitation of labelling to identify different optical signals, the use of input modulated signal to automatically reshape the frequency response of the device. Examples of application are then illustrated to show the validity and generality of the approach, namely a cross-bar interconnect matrix router, a variable bandwidth filter and third order coupled microring filter. Further, the automatic and dynamic generation of the lookup table of add/drop hitless filters operating on a dense wavelength division multiplexing grid is demonstrated. The lookup table achieved with the proposed approach can dynamically update itself to new conditions of the chip or new requirements of operation, such as variations in channel modulation format or perturbation induced by neighboring devices due to a change in their working point.
\end{abstract}

Index Terms - integrated photonics, look up tables, control, calibration, tuning, locking, thermal cross talk, pilot tones. microring resonators, optical filters.

\section{INTRODUCTION}

C ontrol strategies are required in photonic integrated circuits (PICs) to set and hold the working point in a desired state. In a post-production phase, calibration procedures are needed to compensate for fabrication tolerances, to perform quality assessment and to identify faulty devices [1] [2] [3]. This typically means identifying sets of control parameters, such as currents (voltages) driving integrated phase shifters, variable attenuators, optical sources, modulators, etc..., whose values can be stored in a memory of the electronic circuit as lookup tables (LUTs) to be used during the device operation. During operation, tuning procedures are needed to modify on demand the response of reconfigurable PICs [4], [5] [6] as well as to counteract functional drifts due to environmental fluctuations [7]. In this case, control parameters are dynamically set through feedback-loop tuning and locking algorithm and possibly used for a real time update of LUTs.

This work was partially supported by the European Commission through the Horizon 2020 research and innovation program under the Future and Emerging Technologies Open grant agreement Super-pixels (Contract No. 829116).

The authors are with the Department of Electronics, Information and Bioengineering, University Politecnico di Milano, Piazza Leonardo da Vinci, 32, 20133, Milano, Italy. (email: maziyar.milanizadeh@polimi.it)
A possible option to implement calibration and tuning procedures is searching for the control parameters that provide a target frequency response of the PIC. This approach typically matches the requirements for a proof-of-concept demonstration of PIC functionality, but is often time and resource consuming. Measuring the PIC frequency response implies for instance the use of an optical spectrum analyzer while sweeping a tunable laser or using a broadband source. Faster and more efficient methods to automatically tune the PIC response are required, both for calibration and qualification of large volume PICs and for dynamic control of their operation.

Several strategies for automated tuning of PICs have been recently proposed [8], [9] [10], where a suitable cost function is defined for a tuning algorithm aiming for instance at minimizing or maximizing the optical power at specific points of the device, when a number of optical signals are provided at certain input ports. This monitoring can be operated by different kind of integrated detectors, like tap detectors or transparent detectors [11], [12] [13]. However, there are a number of issues to be tackled in order to make automated tuning techniques reported so far more efficient and robust:

- if many actuators need to be controlled, mutual phase coupling (which for thermal tuners is mainly due to thermal crosstalk [14]) can impair the convergence rate of any algorithm or even hinder convergence;

- when multiple optical signals coexist in the same waveguides of a PIC, control algorithm should be able to identify each signal individually in order to tune any device of the PIC to specific signals;

- if the frequency spectrum of the input signals change, for instance because the data rate or the modulation format is modified, the PIC should be able to react and adapt automatically to the new signal spectrum.

In this work we specifically address these issues, proposing key ingredients for implementing simple and robust control and calibration procedures for arbitrary PIC architectures. These concepts are experimentally assessed on different devices fabricated in silicon oxynitride ( $\mathrm{SiON}$ ) and silicon technology. More in details, in Sec. II we discuss a recently developed method, called Thermal Eigenmode Decomposition (TED) 
[15], that enables to cancel out the effects of mutual crosstalk among thermal tuners. In Sec. III we exploit labelling techniques to identify different optical signals in the same PIC and we show how the frequency response of the PIC is tailored accordingly. In Sec. IV we show the automatic reshaping of the frequency response of a PIC to match the power spectral density (PSD) of the input signal. Two further examples of application are then illustrated to show the validity and generality of the afore mentioned ingredients. In Sec. V they are applied to automatically set the bandwidth of a variable bandwidth filter and in Sec. VI they are used to implement the automatic and dynamic generation of the LUT of an add/drop filter operating on a dense wavelength division multiplexing (DWDM) grid. A concluding section summarizes the main results of the paper.

\section{THERMAL CROSS-TALK CANCELLATION}

Any tuning or control technique requires inducing well defined phase shifts in several points of the circuit. Let's assume the phase shifts are provided by heaters but the concept can be extended to phase shifters based on other physical effects. When the heater is activated the heat flow typically generates a thermal crosstalk between adjacent elements that slow down, or even impede, the control process. The cancellation of the
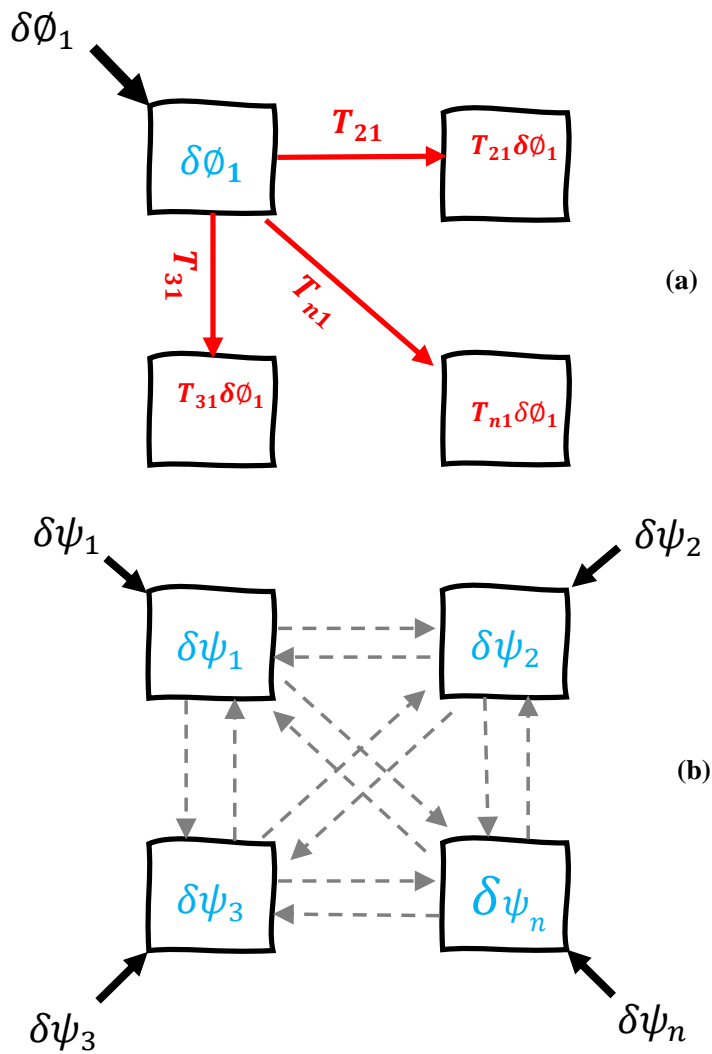

Figure 1: Thermal crosstalk cancellation: (a) Schematic representation of a generic PIC with $n$ tuning elements mutually coupled by thermal crosstalk indicated by coefficients $T_{j k}$. (b) TED concept: the effects of thermal crosstalk (dashed arrows) are mutually cancelled by simultaneously driving all the coupled actuators according to the eigenmodes of the thermally coupled system. The desired phase changes are indicated in blue, while the unwanted phase changes are in red (Color online) thermal crosstalk can be achieved by using the TED approach, that becomes essential as the circuit complexity increases.

To recall the main features of the TED method [9] let us consider Fig. 1(a), showing the schematic of a generic circuit made by 4 controllable elements. In the presence of phase coupling due to thermal crosstalk, when a phase shift $\delta \Phi_{j}$ is thermally introduced in the second element, the neighbor elements are affected by an unwanted phase shift $\delta \Phi_{j} \mathrm{~T}_{\mathrm{kj}}$ where $\mathrm{T}_{\mathrm{kj}}$ are elements of the phase coupling matrix $\mathbf{T}$ [15] whose offdiagonal terms are non-zero in the presence of thermal crosstalk. The matrix $\mathbf{T}$ defines the relation between the vector of desired phase shifts $\delta \boldsymbol{\Phi}$ and the vector of actual phase shift $\delta \widetilde{\boldsymbol{\Phi}}$ as $\delta \widetilde{\boldsymbol{\Phi}}=\mathbf{T} \delta \boldsymbol{\Phi}$.

The TED technique implies the deterministic coordinate transformation $\delta \widetilde{\boldsymbol{\Phi}}=\mathbf{P T}_{\mathrm{D}} \mathbf{P}^{-1} \delta \boldsymbol{\Phi}$, where $\mathbf{P}$ is a matrix whose columns are linearly independent eigenvectors of $\mathbf{T}$ and $\mathbf{T}_{\mathrm{D}}$ is the diagonal matrix containing the corresponding eigenvalues. From this we can define the transformed coordinates $\delta \boldsymbol{\Psi}=$ $\mathbf{P}^{-1} \delta \boldsymbol{\Phi}$, where the system is phase-coupling free, because $\delta \widetilde{\boldsymbol{\Psi}}=\mathbf{T}_{\mathrm{D}} \delta \boldsymbol{\Psi}$. In practice this means that all the actuators of the PIC must be controlled simultaneously as shown in Fig. 1(b) according to appropriate weights, which are based on eigensolution of the thermally coupled system. Once TED is applied, any control algorithm can be implemented by using the transformed coordinates $\boldsymbol{\Psi}$ as phase state variables of the system, including feedback iterative approaches [9], [16] or dithering technique locking approaches [17] [18].

A key point of the TED method is the knowledge of the $\mathbf{T}$ matrix. In principle it could be inferred from thermal simulations. However, this approach is hardly practical for large scale PICs accounting for many heat sources, especially because the overall assembly of the PIC should be considered, including on-chip metal lines, wire bonding, chip submount and ultimately the package itself. Therefore, for an accurate estimation of the $\mathbf{T}$ matrix, alternative strategies should be adopted, which should be preferably applicable to arbitrary PIC architectures.

In the following, we consider three different solutions to evaluate the thermal crosstalk with different accuracy:

1) heuristic (lowest accuracy): an estimation of thermal cross talk is inferred by simply considering the topology of the circuit;

2) optical measurement (highest accuracy, not always practicable): thermal crosstalk is directly estimated by measuring the unwanted wavelength shift of neighbor devices;

3) electrical measurement (good accuracy, always practicable): thermal crosstalk is indirectly estimated by measuring the change of the electric resistance of neighbor heaters used as temperature probes.

The evaluation of the $\mathbf{T}$ matrix is presented in Section IV and the impact of its accuracy on TED based tuning algorithm discussed. It is worth to mention that $\mathbf{T}$ depends only on the device architecture and not on the specific operation and, as any transfer function, must be evaluated once, independently on the circuit working point. 


\section{SignAL LABELLING}

The second considered best practice is the labelling of the signals to be processed with weak pilot tones [19]. These labels are implemented through a shallow modulation on the channel which can be distinguished at the detector. As an example, if two channels ( $\mathrm{Ch} 1$ and $\mathrm{Ch} 2)$ are labeled by modulations with frequencies $f_{i}$ and $f_{e}$ respectively, at the detector they can be distinguished by lock-in demodulation of the corresponding frequency without affecting the quality of the signal [11].

As a case analysis, we consider a $2 \times 2$ cross-bar interconnect of microring resonators (MRR). This device is used to route the inputs signals, either a single channel or a comb of WDM channels. Figure 2 (a) shows a top view microphotograph of the device, which was fabricated in a high-index-contrast silicon oxynitride ( $\mathrm{SiON}$ ) photonic platform [20]. The SiON channel waveguide $(2.2 \mu \mathrm{m} \times 2.2 \mu \mathrm{m})$ has a refractive index contrast of $4.4 \%$ and the MRR resonators have a free spectral range of 50 GHz. The round-trip phase of each MRR of the filter can be individually controlled by means of metallic heater deposited on top of the waveguide uppercladding. Phase perturbations were intentionally introduced in every MRR of the PIC by applying random errors in the voltages driving the heaters around their optimum tuning point simulating the circuit variability. The measured transmission from WP2 port to SP2 port of the filter is shown in Fig. 2(b).

A TED-based gradient descent algorithm employing the transformed coordinates $\delta \boldsymbol{\Psi}$ was used to automatically (a)

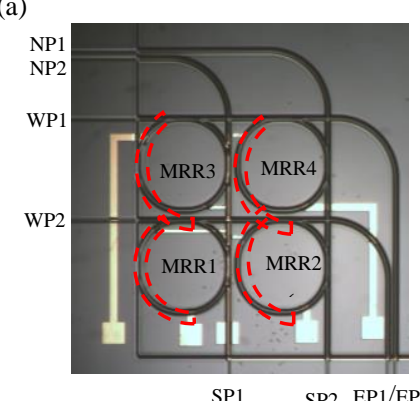

(c)

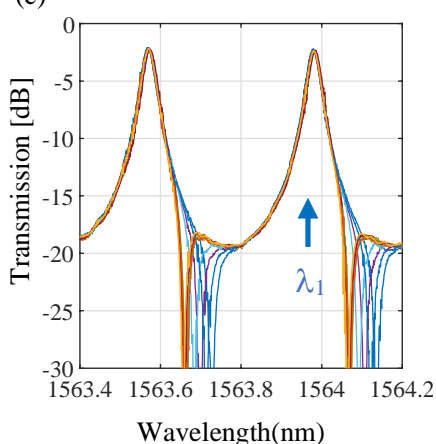

(b)

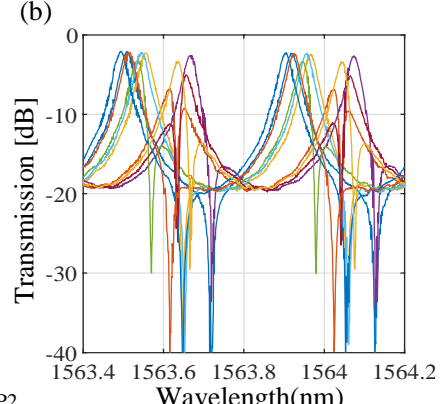

(d)

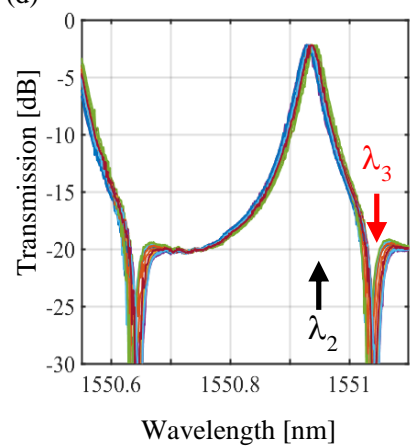

Figure 2: (a) Top view photograph of a 2x2 MRR cross-bar interconnect fabricated in SiON technology. The red dashed lines indicate the position of the heaters integrated on the MRRs. Measured transmission from WP2 port to SP2 port (b) for 10 randomly perturbed configurations induced by using thermal phase shifters, and after automated tuning (c) to a signal at $\lambda_{1}=1563.98 \mathrm{~nm}$, and (d) to signals at $\lambda_{2}=1550.93$ and $\lambda_{3}=1551.03 \mathrm{~nm}$. configure the circuit in order to set an optical path from input WP2 to output SP2 at a single CW signal at wavelength $\lambda_{1}=$ $1563.98 \mathrm{~nm}$. Results in Fig. 2(c) show that regardless of the initial perturbation, the filter was effectively tuned to maximum transmission at the desired wavelength, which is achieved by correctly setting the resonant frequency of MRR2. It should be noted that the final transfer functions of the circuit overlap only around the target wavelength $\lambda_{1}$, and periodically every free spectral range (FSR), but are not exactly the same.

This effect is expected to happen at wavelengths where no cost function is defined and, depending on the degrees of freedom of the system, the frequency response of the PIC may converge to random solutions (in this specific case the variability of the frequency response is related to the unconstrained position of the resonance of MRR1 that generates a notch adjacent to MRR2 resonance). To manage this problem, the same algorithm was then used to route two channels, namely $\lambda_{2}=1550.93 \mathrm{~nm}$ and $\lambda_{3}=1551.03 \mathrm{~nm}$, which were simultaneously injected at input port WP2, to output ports SP2 and SP1, respectively. To this aim, the two signals were labeled each via a shallow modulation tone, with a modulation depth of $<8 \%$ and a frequency $\mathrm{f}_{1}=11 \mathrm{kHz}$ and $\mathrm{f}_{2}=17 \mathrm{kHz}$. The tuning algorithm was targeted to maximize the ratio between the amplitude of tones at the port SP2, that is to maximize the isolation between the two signals. Figure 2(d) shows that the frequency responses of the MRR router, which is constrained both at the peak of the transmission curve $\left(\lambda_{2}\right)$ and at the notch $\left(\lambda_{3}\right)$, nicely overlap across the entire wavelength range.

\section{THERMAL CROSSTALK MATRIX EVALUATION AND PERFORMANCE}

The evaluation of the thermal crosstalk $\mathbf{T}$ matrix is here explained and its impact on the effectiveness on TED based technique is investigated. The analysis is performed on the MRR based cross-bar router of Fig. 2.

According to Sec. II, with a heuristic approach we assume that all the actuators introduce equal thermal crosstalk on surrounding MRRs. The corresponding phase coupling matrix $\mathbf{T}$ is hence

$$
\mathbf{T}=\left(\begin{array}{llll}
1 & \mu & \mu & \mu \\
\mu & 1 & \mu & \mu \\
\mu & \mu & 1 & \mu \\
\mu & \mu & \mu & 1
\end{array}\right)
$$

where $\mu=0.1$ is a good estimation, done experimentally of the average thermal crosstalk. Each column of the $\mathbf{P}$ matrix is an eigenvector of the matrix $\mathbf{T}$ in eq. (1). The matrices $\mathbf{P}^{-1}$ and $\mathbf{T}_{\mathbf{D}}$ are reported in Appendix. The phase change along the orthogonal transformed coordinates can be calculated as $\delta \boldsymbol{\Psi}=$ $\mathbf{P}^{-1} \delta \boldsymbol{\Phi}$. Using these orthogonal vectors, uncoupled modifications can be applied by tuning algorithm (Fig. 1(b)).

To provide a metric for evaluating the effectiveness of thermal crosstalk compensation, Fig. 3(a) shows the convergence curve of the TED-based gradient descent algorithm, that is the optical power at the output port SP2 versus the number of iterations. Assuming a heuristic T matrix, the 
average number of iterations that are required to set the optical path is about 40, as shown by the green dashed curve in Fig. 3(a). As a benchmark (black diamonds), we consider the case where TED is not used, that is the MRRs are individually controlled by applying phase shifts $\delta \boldsymbol{\Phi}$. Because of thermal crosstalk, we observe an extremely slow convergence and, depending on the initial perturbed state of the PIC, the algorithm may even not converge to the target response.

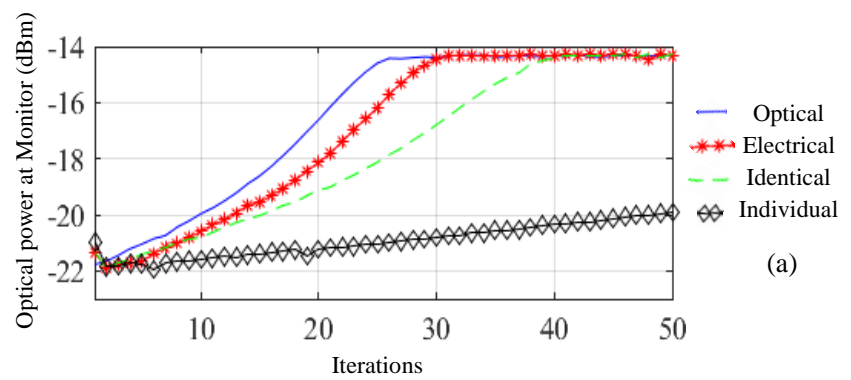

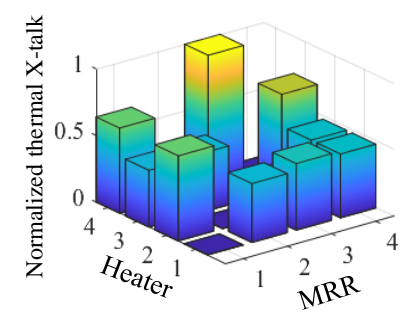

(b)

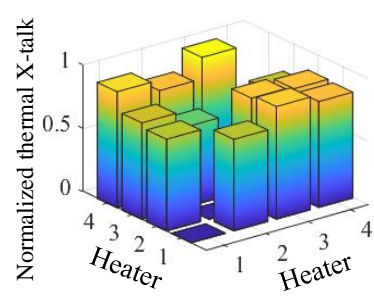

(c)
Figure 3: (a) Convergence rate of TED-based tuning of the 2x2 MRR crossbar interconnect of Fig. 2a when the off-diagonal terms of the phase coupling matrix $\mathbf{T}$ are assumed to be identical (green dashed), when they are estimated from electrical measurements (red asterisks), from optical measurements (blue solid). Black diamonds show the converge rate when MRRs are individually tuned (no-TED). (b) Optically and (c) electrically measured off-diagonal terms of the phase coupling matrix $\mathbf{T}$ normalized to the maximum value.

Faster convergence is achieved when the off-diagonal (crosstalk) terms of the phase coupling matrix $\mathbf{T}$ are closer to the actual values. An exact evaluation is achievable in the considered PIC because the coefficients of the $\mathbf{T}$ matrix can be directly estimated through optical measurements. In fact, since all the output ports of all the MRRs are accessible, we measured the cross-induced shift of each MRRs resonances when the different heaters are sequentially switched on. Figure 3(b) shows the optically-measured off-diagonal terms of the phase coupling matrix $\mathbf{T}$. More specifically each pillar provides the induced crosstalk by each heater on the surrounding MRRs as the wavelength shift of that MRR to the source MRR (heater) wavelength shift (normalized to the maximum crosstalk). It should be noted that not only the off-diagonal terms are not equal [this being rather expected and in contrast with the heuristic assumption made in eq. (1)], but they are also not symmetric. This asymmetry is justified by considering that the heaters are not as long as the MRR circumference, but they only cover a portion of each MRR. As a result, the distance of heater1 (integrated in MRR1) from the waveguide of MRR2 is larger than the distance of heater2 (integrated on MRR2) from the waveguide of MRR1. As shown by the solid blue line in Fig. 3 (a), by using the optically measured value of the thermal crosstalk the $\mathbf{T}$ matrix, the average number of iterations that are required to set the optical path is about 25 , thus proving faster convergence.

However, a direct optical measurement of thermal crosstalk is not possible in most cases, because it requires the possibility to optically access the output ports of each tunable element. A good estimation of the $\mathbf{T}$ matrix can be achieved electrically, by measuring the temperature-induced change of the resistance of metallic probes located in suitable spots of the PICs. To this aim, we can advantageously use the heater structures themselves, which can be used also as temperature sensors to map thermal crosstalk across the photonic chip. Figure 3(c) shows the electrically-estimated off-diagonal terms of the phase coupling matrix $\mathbf{T}$. In this case, each pillar provides the resistance change of each heater to the resistance change of the perturbing heater (normalized to the maximum crosstalk). In the considered case the heaters are realized as a thin layer of Nickel-Chromium with a nominal resistance of $562 \mathrm{Ohm}$ and a temperature dependence of $\alpha=0.000341 /{ }^{\circ} \mathrm{C}$ in $\Delta R / R=$ $\alpha . \Delta T$. We use lock-in detection scheme with resolution of 30 $\mathrm{ppm}$ which for our heaters meant variation of $17 \mathrm{mOhm}$. With this setup we can measure 0.1 degree of change in our sensors.

It should be noted that the electrically measured $\mathbf{T}$ matrix differs from the optically estimated one of Fig. 3(b). This difference can be justified by considering that the electrically measured $\mathbf{T}$ matrix provides the thermal crosstalk at specific points where probe heaters are placed (heater-to-heater), while the optically measured $\mathbf{T}$ matrix provides the exact value of thermal crosstalk integrated over the optical ring path (heaterto-ring). As shown by the red asterisks in Fig. 3(a), by using the electrically-estimated $\mathbf{T}$ matrix in the TED method, convergence is achieved with a number of iterations comprised between the optimum (optically-estimated $\mathbf{T}$ matrix) and the heuristic $\mathbf{T}$ matrix. As a main advantage, an electrical measurement of thermal crosstalk can be performed in arbitrary PIC architectures, by using already existing metal structures (e.g. heaters) and/or by adding metal probes in specific points of the PIC to get a detailed mapping of the temperature gradient across the photonic chip.

\section{Automated TUNing to Optical Signals}

Traditionally, PIC tuning is implemented targeting a specific frequency response. This approach can be valuable for testing, and pre-calibration procedures, but it is not practical for automated tuning of the PIC during its operation because it is resource and time consuming. For instance, it may require a tunable source and/or a spectrum analyzer to monitor the response at wavelengths of interest. Likewise, time domain approaches, based on the measurement of the bit error rate (BER) or eye diagram distortion, require considerable load of signal processing as well as electrical power consumption. In any case both approaches are hardly practical when the device is in use. 
Here we discuss the automated tuning of the PIC response according to the power spectral density (PSD) of the optical signal provided at the input. Basically, we define a simple cost function, which could essentially be the maximization (minimization) of the signal power transmitted though the PIC, from a specific input port to a specific output port. This power can be simply measured either at an end port of a device or through the integrated monitors (tap detectors [3] or transparent detectors (Clipp [11]). If multiple signals (channels) coexist in the same optical waveguide of the chip, labelling strategies (tones) can be used to discriminate the power of a given channel regardless from the presence of other channels [19]. We can anticipate that, since the filter is tuned looking at a specific channel, by changing the central wavelength, the bandwidth or the modulation format of the channel, that is its PSD, the PIC frequency response will be automatically tailored to optimize the filter metric on that channel. Therefore, even though the circuit architecture is not supposed to have a pronounced reconfigurability (e.g. bandwidth tunability), some frequency reshaping effect will be dictated depending on the input signal.

To demonstrate this concept, we consider the third order coupled resonator MRR filter of Fig. 4(a), which was fabricated in the same SiON photonic platform as the device of Fig. 2. This index contrast enables the realization of MRRs with an FSR of up to $100 \mathrm{GHz}$ with negligible bending loss. Initial phase perturbations were intentionally introduced in every MRR of the filter by applying random deviations in the voltages driving the heaters around their optimum tuning point. Figure 4(b) shows the measured Through and Drop port transmission of the perturbed filter when the frequency spread of the MRR resonances is as large as $100 \mathrm{pm}(12.5 \mathrm{GHz})$.

As a first example, we used an intensity modulated $5 \mathrm{Gbit} / \mathrm{s}$ OOK input signal with a carrier wavelength $\lambda_{1}=1565.470 \mathrm{~nm}$, whose bandwidth $\mathrm{B}_{\mathrm{s}}=5 \mathrm{GHz}$ well matches the nominal filter bandwidth $\mathrm{B}_{\mathrm{f}}=6.5 \mathrm{GHz}$. The optical power is measured through an external photodiode and transmitted to a PC where a TED-based gradient-descent tuning algorithm (details in [15] and Section II) was employed to minimize the output power transmitted at the Through port. As a metric for the performance of the automated tuning we assume the Through port isolation $I_{\mathrm{B}}$ averaged across the bandwidth $B_{s}$ of the signal (centered around $\lambda_{1}$ ). The transfer functions of the tuned configurations of filter for Through and Drop port are shown in Fig. 4(c). The dashed box indicates the wavelength range $B_{s}$ occupied by the signal. The overlap among all the measured curves, regardless of the initial perturbed state, demonstrates the accuracy of the tuning procedure, guaranteeing an isolation $I_{5 \mathrm{GHz}}=15 \mathrm{~dB}$. It should be noted that in this condition $\left(B_{\mathrm{s}} \approx B_{\mathrm{f}}\right)$, the frequency response of the filter is the nominal one, that is achieved when the three MRRs resonances are aligned.

Starting from the same random filter configurations as in Fig. 4(b), we reduced the data rate of the signal to $2.5 \mathrm{Gbit} / \mathrm{s}$, thus halving the bandwidth $\mathrm{B}_{\mathrm{s}}$ smaller than $5 \mathrm{GHz}$. As shown in Fig. $4(d)$, the filter is now automatically tuned to a shape that is much different from the one shown in Fig. 4(c). In this case, where $B_{\mathrm{s}}<B_{\mathrm{f}}$, the three MRRs are not resonating at the same

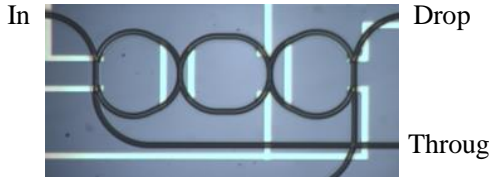

(a)

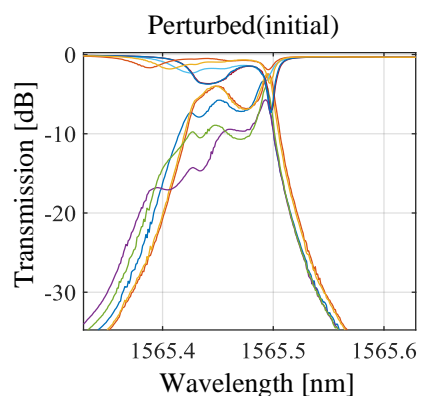

(b)

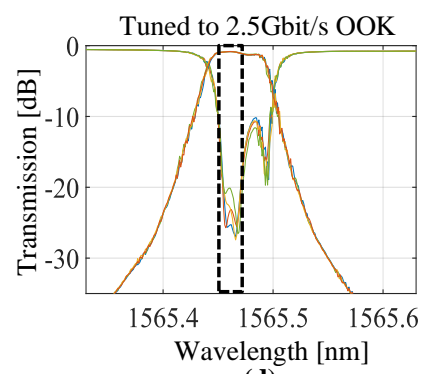

(d)

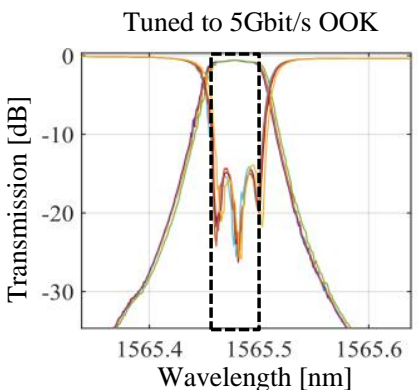

(c)

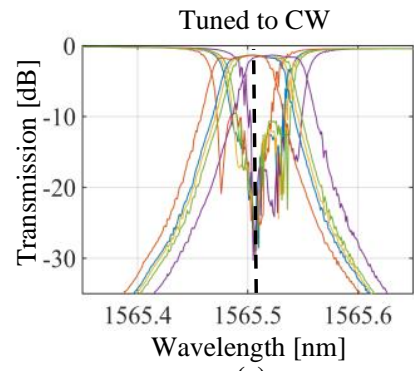

(e)

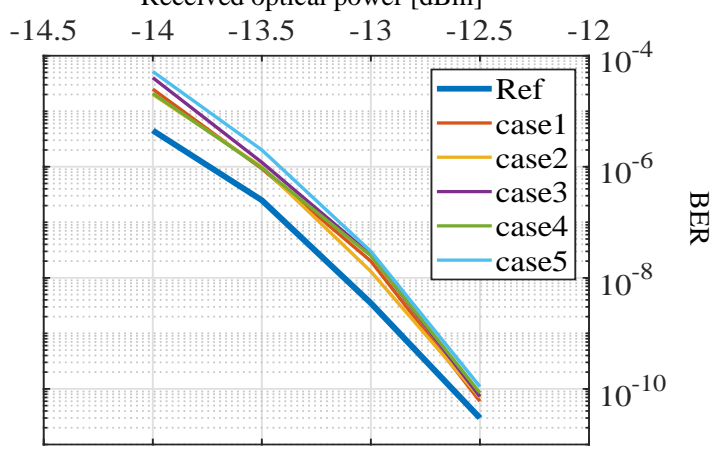

(f)

Figure 4: Tuning to optical signals: (a) top view photograph of the $3^{\text {rd }}$ order MRR SiON filter. Measured frequency response at Drop and Through ports for (b) intentionally random initial states and after automated tuning using as an input signal (placed at position of dashed box): (c) a $5 \mathrm{Gbit} / \mathrm{s}$ OOK channel, (d) a 2.5Gbit/s OOK channel, and (e) a CW laser source (no modulation). (f) BER measurements of a $2.5 \mathrm{Gbit} / \mathrm{s}$ OOK channel transmitted at the drop port of the automatically tuned filter. The blue curve is the reference BER obtained at Through port when the filter is detuned from the signal.

wavelength, but this filter shape provides higher isolation $\left(I_{2.5 \mathrm{GHz}}>20 \mathrm{~dB}\right)$ across the signal bandwidth (dashed box).

The automatic tailoring of the frequency response of the filter according to the PSD of the input signal is even more pronounced if the data modulation is switched off. As shown in Fig. 4(f), the frequency responses achieved using a CW source at a wavelength of $1565.5 \mathrm{~nm}$ overlap well only at the wavelength of the optical signal, where an isolation $I_{\mathrm{CW}}=30$ $\mathrm{dB}$ is achieved. Yet, the automated tuning does not bring the 
filter to a unique filter shape. This is strongly related to the fact that $B_{\mathrm{s}} \ll<B_{\mathrm{f}}$. In fact, at wavelengths where the signal PSD vanishes the tuning algorithm is unconstrained and may converges to random filter shapes.

To demonstrate that the automatically tuned frequency responses of the filters are equivalent for the considered input channel, we measured the bit error rate (BER) of the $2.5 \mathrm{Gbit} / \mathrm{s}$ OOK signal used in Fig. 4(d) when it is transmitted to the Drop port of the filter. BER curves in Fig. 4(f) were achieved from 5 different drop-port filter configurations, after convergence of the tuning algorithm from different initial states of the filter. All the considered cases share the same BER performance, with less than a half $\mathrm{dB}$ power penalty with respect to the reference BER obtained by off-band transmission at the Through port.

\section{APPLICATION I: AUtOMATED AdAPTIVE TUNING OF RECONFIGURABLE FILTERS}

The automatic adaptation of the filter shape to the PSD of the input signal is a general concept that can be exploited in arbitrary PICs, especially in the ones with a reconfigurable frequency response. As an example, let us consider the PIC of Fig. 5(a), consisting of unbalanced Mach-Zehnder Interferometer (MZI) loaded with two MRR all-pass filters. This architecture implements a tunable bandwidth filter, since the bandwidth can be controlled by tuning the relative positions of the MRR resonances and the phase unbalance between the two arms of the MZI [21]. The MZI has $3 \mathrm{~dB}$ couplers, while the power coupling between the MRRs and the bus waveguide is 0.66. With these parameters the bandwidth of the filter, whose FSR is $25 \mathrm{GHz}$, can be tuned from $0.25 \mathrm{FSR}(7 \mathrm{GHz})$ to 0.7FSR $(17 \mathrm{GHz})$.

Different initial perturbations were intentionally introduced as random errors in the voltages driving the three heaters (2 MRRs and MZI) around their optimum tuning point. Figure 4(b) shows the frequency response of bar and cross port of perturbed filters when up to $20 \%$ phase errors are introduces. The PIC was automatically tuned by using a TED-based gradient-descent tuning algorithm to minimize the optical power at cross port of filter. As input signal we used a channel with $10 \mathrm{Gbit} / \mathrm{s}$ OOK modulation at the wavelength of 1563.87 $\mathrm{nm}$. Since in this device a direct optical measurement of the phase coupling matrix $\mathbf{T}$ is not possible, due to the lack of accessible monitor ports at the output of each tunable device, we performed electrical measurement of thermal crosstalk, as shown in Sec. IV.

The frequency response of the tuned filters at convergence is shown in Fig. 5(c) for cross port (red lines) and bar port (blue lines). Results demonstrate that independently of the initial perturbed state of the filter, a very good overlap of the filter shapes at convergence is achieved. Since the algorithm aims at minimizing the signal transmission at the cross port, at this port the isolation averaged across the bandwidth $B_{s}=10 \mathrm{GHz}$ of the signal is the maximum the filter can provide, that is higher than $27 \mathrm{~dB}$. In contrast, the isolation at the bar port is lower $(<20$ $\mathrm{dB}$ ), not because of some filter imperfection, but because this parameter is not considered in the cost function of the

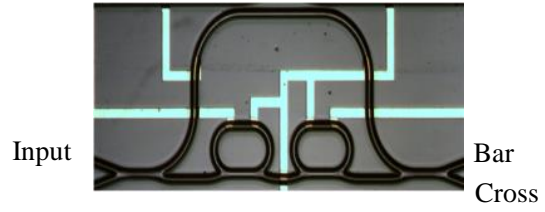

(a)

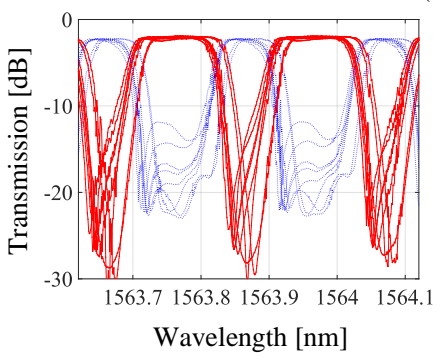

(b)

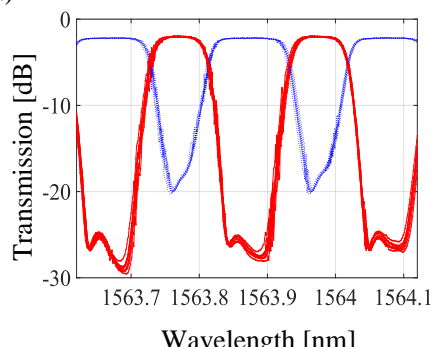

(c)
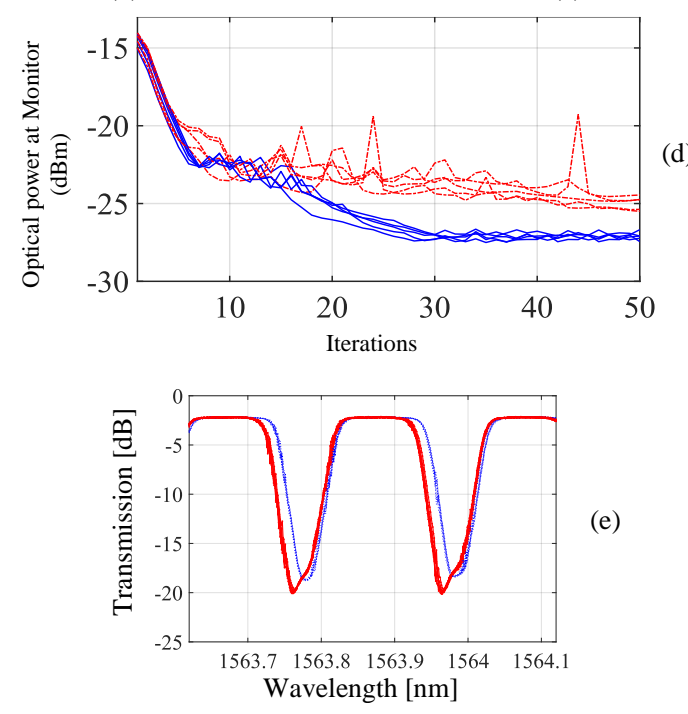

(e)

Figure 5: (a) Top view photograph of a SiON MZI loaded with 2 MRRs implementing a tunable bandwidth filter. Measured transmission at bar and cross port in (b) intentionally perturbed states and (c) after automated tuning to a $10 \mathrm{Gbit} / \mathrm{s}$ signal at $1563.87 \mathrm{~nm}$. (d) Comparison of convergence performance of the TED-based tuning algorithm using electrically measured (blue) and heuristic (red) T matrix (e) Automated adaptation of the filter spectral response (bar port) when the signal bandwidth is $B_{s}=10$ $\mathrm{GHz}$ (red) and $B_{s}=28 \mathrm{GHz}$ (blue).

algorithm. Therefore, the passbands of the filter at the two ports are different, the bar port one $(14.5 \mathrm{GHz})$ being wider than the passband one $(9.5 \mathrm{GHz})$. To achieve a symmetric shape at the two ports, two channels spaced by FSR/2 and labelled with different tones should be simultaneously transmitted through the filter and a suitable cost function should be defined for the tuning algorithm.

The convergence of the tuning is shown in Figure 5(d) where the optical power at the monitored cross-port versus the number of iterations is shown. Starting from the same perturbed state of the filter, solid blue lines represent the convergence curves when the electrically measured $\mathbf{T}$ matrix is used in the TED method, while dashed red lines refer to the case of the heuristic T matrix of eq. (1) with identical off-diagonal terms $\mu=0.02$. In both cases convergence is achieved, but fine optimization at 
high isolation degree is faster and more robust when thermal crosstalk is better compensated.

Exploiting the tuning on the signal as explained in Section V, the filter adapts to the signal bandwidth. In Figure 5 (e) frequency response of ten tuned filters are presented for the case of DP-QPSK modulated channel with $B_{s}=28 \mathrm{GHz}$ in blue and channel with $B_{s}=10 \mathrm{GHz}$ in red in their bar port. Filters tuned to wider channel have broader notches in their cross port frequency response to minimize better the signal (which is not shown in figures), therefore their bar port frequency response demonstrate wider pass bandwidth which is easier to observe (compared) and is presented in Fig 5(e).

\section{APPLICATION II: AUTOMATIC LUT GENERATION}

The control and calibration techniques described in the previous sections enable the automatic tuning and locking of a device to the desired working point, even in an adaptive way. However, when the device is far away from the target functional state because it is in the initial state or a consistent variation of the working point is required, the device usually needs to be led to work in the desired condition using pre-determined values for its control parameters which are stored in a LUT. As discussed in Sec. I, building up a LUT is usually very time consuming, because the device is in an unknown state and specific procedures need to be followed which are heavily dependent on the circuit architecture, on the working conditions, and on the initial state of the circuit.

An efficient process to generate the LUT can exploit the automatic tuning process using the thermal crosstalk free tuning algorithms, the modulated channel as feedback signal and possibly the pilot tones to discriminate the desired signal. Once the tuning reaches the convergence, the final currents/voltages of the actuators are stored in the LUT. These LUTs can be even dynamically updated during the operation of PIC to match the new requirements of the system, for example when the channel modulation format or the thermal crosstalk form neighboring devices changes.

We applied these concepts to the automated generation of a LUT for an add/drop filter operating on a dense wavelength division multiplexing (DWDM) grid. The block diagram of the considered device along with the control loop scheme is shown in Fig. 6(a). The filter unit is a $3^{\text {rd }}$ order coupled MRR filter, which is designed to have $1 \mathrm{THz}(8 \mathrm{~nm}) \mathrm{FSR}, 40 \mathrm{GHz} 3 \mathrm{~dB}$ bandwidth, and $20 \mathrm{~dB}$ in-band isolation. A thermally tunable unbalanced MZI is employed as a tunable switch to provide a hitless functionality [22]. A second balanced MZI is integrated at the Add port and it is used as a channel labeler to superimpose to the added channel a weak pilot tone in order to make it distinguishable from the other WDM channels incoming form the Input port. A transparent detector (CLIPP) [11] is integrated at the Drop port in order to read the residual power of the added labelled signal, thus providing an error signal to the controller without affecting the dropped signal. More specifically, the CLIPP reads the label amplitude through a lock-in detection scheme [19]. An FPGA reads the CLIPP measurements while controlling the heaters alongside the filter. A microscopic
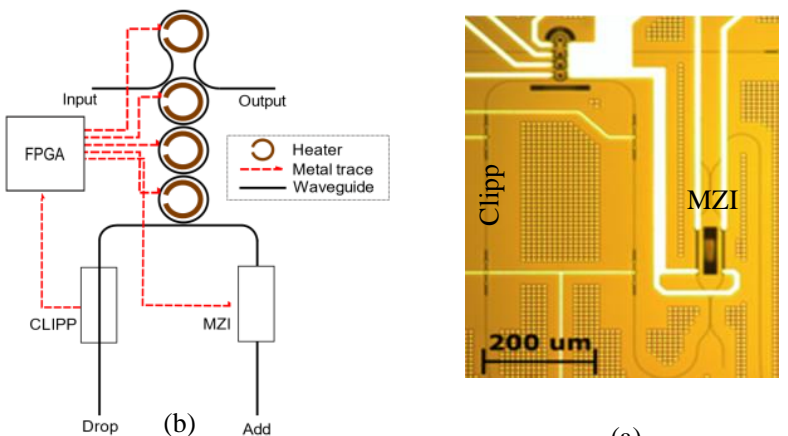

(a)

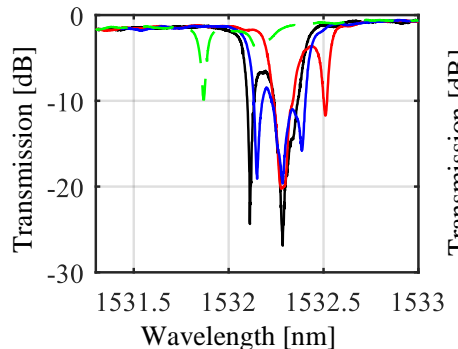

(c)

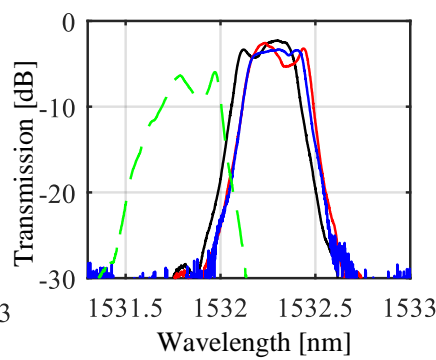

(d)
Figure 6: (a) Functional block diagram of a reconfigurable hitless filter along with the relevant control elements adopted to demonstrate fine tuning and automatic look up table creation (b) Top-view photograph of overall filtering unit realized on a silicon chip, including monitoring CLIPP detector and on-chip MZI labeler to mark the added signal. Through (c) and Drop port (d) frequency response of filter starting from same initial conditions (natural response in green-dashed) and tuned by using a 100Gbit/s DP-QPSK signal (blue), a $10 \mathrm{Gbit} / \mathrm{s}$ OOK signal (red) and CW source (black).

picture of the photonic chip fabricated on a silicon photonic platform is shown in Fig. 6(b). More details on the filter architecture cha be found in [22].

Starting from the natural frequency response of the filter (green-dashed curves in Fig. 6(c) for Through port and in Fig. 6(d) for Drop port response), a modulated channel at 1532.25 $\mathrm{nm}$ is launched in the Add port and is labelled by the integrated MZI $(11 \mathrm{kHz}$, modulation depth $<8 \%)$. Figures $6(\mathrm{c}, \mathrm{d})$ present the frequency response of the tuned filter, when the input signal is modulated according to a $100 \mathrm{Gbit} / \mathrm{s}$ QPSK (blue curve), 10 Gbit/s OOK (red curve) and when no modulation is applied (black curve). The frequency response of filter is reshaped to match the PSD of the employed signal. The narrower filter bandwidth obtained with the $\mathrm{CW}$ channel allows to achieve higher in-band isolation $\left(I_{\mathrm{CW}}=27 \mathrm{~dB}\right)$ while for $100 \mathrm{Gbit} / \mathrm{s}$ QPSK channel, a larger bandwidth and a corresponding lower in-isolation across the $28 \mathrm{GHz}$ signal bandwidth $\left(I_{100 \mathrm{~Gb}}=18 \mathrm{~dB}\right)$ is achieved. Ripples of filter tuned to $10 \mathrm{Gbit} / \mathrm{s}$ OOK transmitter are out of the bandwidth of the signal therefore not perturbing the quality of the channel.

Once the filter has been tuned at the first channel of the WDM grid, the procedure can be iterated to create the LUT over the entire operative wavelength range. We used a commercial $100 \mathrm{Gbit} / \mathrm{s}$ transmitter whose carrier wavelength, data rate and modulation format can be selected and a LUTs matching an 
arbitrary sequence of optical channels has been created. In our setup the FPGA selects the carrier wavelength of the modulated input signal fed to the Add port of the filter and execute automatically the tuning algorithm to find the tuned parameters of the filter for that channel. This is repeated till the table is filled up based on the performance requirements of the filter along the frequency band.

To illustrate this approach, let us consider an architecture including a pair of neighbor add-drop filters as in the scheme Fig. 7(a). Figure 7(b) shows the tuning of filter I when eight different channels with $100 \mathrm{Gbit} / \mathrm{s}$ QPSK modulation and 50 $\mathrm{GHz}$ frequency spacing are sequentially provided at the input. The Drop and Through port transfer functions of the filter controlled by this automatically created lookup table are very well in line with the reference shape shown in Figs. 6(c)-(d). To check if the LUT can be created and dynamically updated in presence of perturbations, we used the neighbor filter II, which is connected to the same bus waveguide of filter I. The tuning of filter II is performed by using a $10 \mathrm{Gbit} / \mathrm{s}$ OOK modulated channel when filter I is operative (that is when it is tuned to one of the eight $100 \mathrm{Gbit} / \mathrm{s}$ channels). Figure 7(c) shows that the frequency response of filter II is automatically shaped to match the expected channel bandwidth [see 6(c)-(d)], regardless of the tuning state of the adjacent filter I. In other words, a LUT for filter II (and vice-versa) can be created and updated regardless of the thermal crosstalk from surrounding devices.

As a final demonstration let us consider the scenario of elastic networks [23], where optical channels can change their bandwidth (modulation format or bit rate) or even wavelength placement during operation. By using our tuning-to-signal approach, the LUT is automatically updated for this new status. For instance, starting from the configuration of Fig. 7(b) for filter I, let us assume that channels number 2, 5 and 7 change their modulation scheme from $100 \mathrm{Gbit} / \mathrm{s}$ QPSK to $10 \mathrm{Gbit} / \mathrm{s}$ OOK and channel 3 remove its modulation and transmit only the carrier [(channel numbering is here defined from left to right on Fig. 7(b)]. Figure 7(d) shows for Drop and Through ports transfer function according to the modified channel grid, where the black dashed line represent the three $10 \mathrm{Gbit} / \mathrm{s}$ OOK channels, the red line is the filter tuned to a $\mathrm{CW}$ signal, and the blue lines are the original four $100 \mathrm{Gbit} / \mathrm{s}$ QPSK of Fig. 7(c).

\section{CONCLUSION}

We presented several strategies for implementing simple, effective and robust control and calibration procedures for arbitrary PIC architectures.

The effect of mutual crosstalk induced by thermal tuners is cancelled by using as control parameters the eigenvectors of the thermally coupled system (TED technique). The TED method enables to speed up the convergence of generic automated tuning algorithms and is more robust against instabilities and convergence failures. The best performance of TED-based algorithms is achieved when the phase coupling matrix $\mathbf{T}$ describing thermal crosstalk is exactly known, which is hardly achievable in most cases. However, just slightly sub-optimal performance is observed with an approximate estimation of the
T matrix, which can be easily achieved electrically by exploiting integrated heaters as temperature sensors.

Tuning procedures are advantageously carried out by defining a suitable cost function on the optical signal(s) at the output of the PIC without targeting a predefined frequency response. This enables to steer the PIC to a state that optimizes its operation on those specific channels. In order to make it possible when several signals coexist in the same PIC, each signal is labelled though weak modulation tones in such a way that it can be monitored independently form the others by a lock-in detection. Applying this concept to a $2 \times 2$ cross-bar interconnect of MRRs we showed that optical paths across the
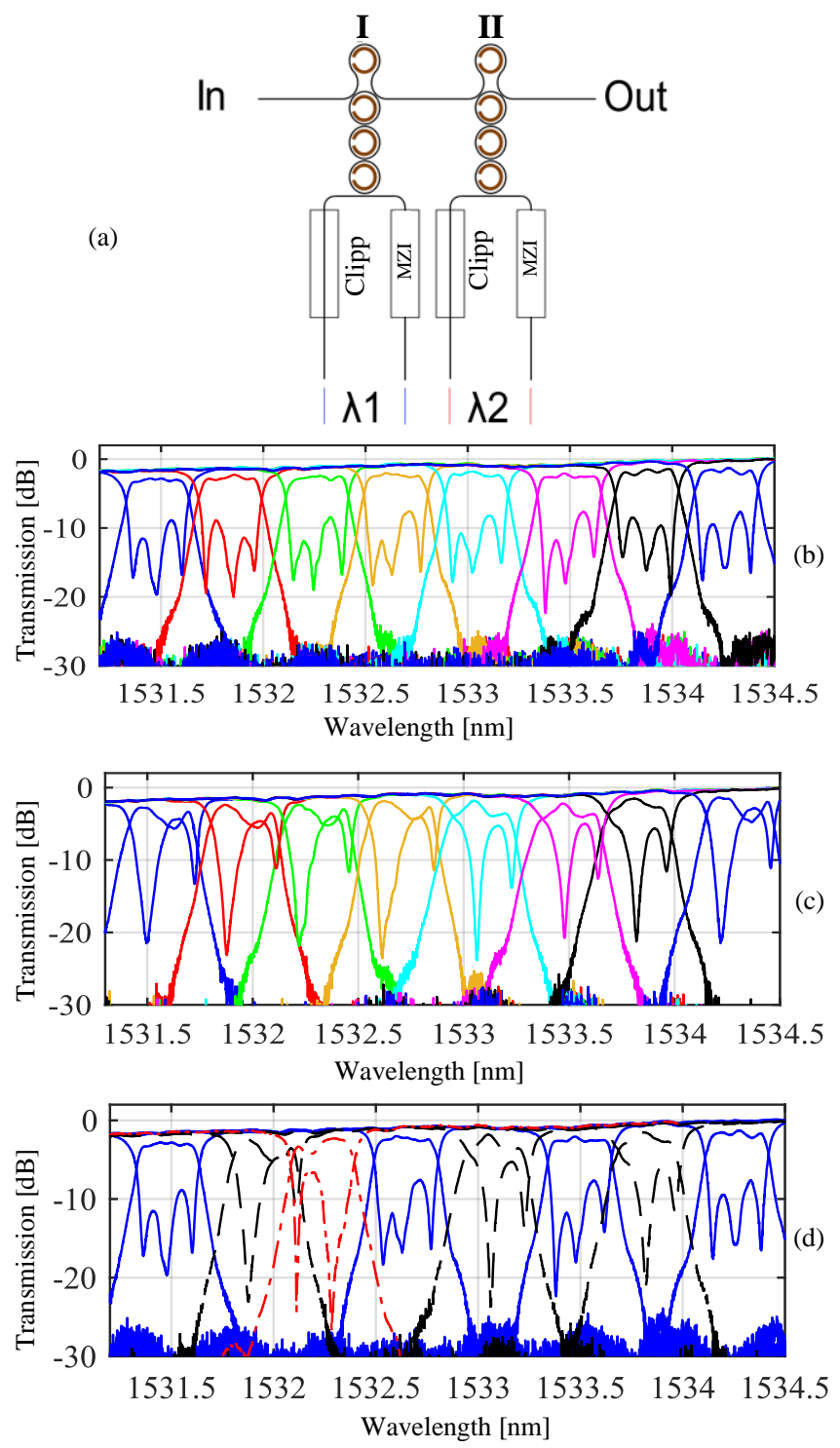

Figure 7: (a) Functional block diagram of two reconfigurable add-drop filters sharing the same bus waveguide, demonstrating a bi-channel add/drop block (b) Through and Drop port frequency response of filter I with automatically created lookup table for ten $100 \mathrm{Gbit} / \mathrm{s}$ DP-QPSK channels spaced by $50 \mathrm{GHz}$. (c) Through and Drop port transfer function of filter II (while filter I is operative) from automatically generated lookup table for ten $10 \mathrm{Gbit} / \mathrm{s}$ OOK channels spaced 50GHz apart. (d) Drop and Through port transfer function of filter I according to a flexible channel grid with three $10 \mathrm{Gbit} / \mathrm{s}$ OOK channels (black dashed), one CW signal (red dash) and four $100 \mathrm{Gbit} / \mathrm{s}$ QPSK (blue solid). 
PIC are correctly set when the cost function can discriminate the optical power of each signal.

If the spectral characteristics of the signal change, for instance its bandwidth, the control parameters driving the PIC actuators are automatically modified to re-optimize the cost function on the new set of input signals. We showed that the frequency response of a third order coupled MRR filter, which is not supposed to be a tunable bandwidth device, is significantly reshaped when the bandwidth of the input signal is reduced (from $5 \mathrm{GHz}$ to $\mathrm{CW}$ ), in order to maximize the isolation across the signal bandwidth. This feature is of particular interest for programmable filters, such as the variable bandwidth filter shown in Sec. IV where, instead of using predetermined values of the control parameters to obtain a specific response, automated tuning of the filter to the optical signals try to find the best compromise for all the present channels, according to the defined cost function.

All the control strategies presented in this work were employed to the calibration and control of an add/drop hitless filter operating on a dense wavelength division multiplexing (DWDM) grid [22]. In particular we successfully demonstrated that these procedures can be used to automatically generate and dynamically update the lookup table of the filter when it is operated on a system with flexible bandwidth channels from 10 $\mathrm{Gbit} / \mathrm{s}$ to $100 \mathrm{Gbit} / \mathrm{s}$.

Finally, these techniques, which are here applied to MRRbased architectures, can be employed to generic reconfigurable PIC architectures with an arbitrary topology and implementing different functions, such as MZI switch matrices [24] [25] and programmable photonic processors [26] [27].

\section{APPENDIX}

As supplementary information, in this appendix we provide the numerical values of the phase coupling matrix $\mathbf{T}$ of the $2 \times 2$ MRR cross-bar interconnect of Fig. 2, as evaluated with the three different approaches presented in Sec. IV. To better highlight the mutual crosstalk level, we focus on the relative values of the off-diagonal terms of the $\mathbf{T}$ matrix, which are collected in a modified matrix $\mathbf{T}_{\mathrm{m}}$, which is achieved by zeroing the main diagonal of the $\mathbf{T}$ matrix and normalizing to the maximum off-diagonal term. The elements of the optically and electrically measured $\mathbf{T}_{\mathrm{m}}$ matrix are indeed shown in Fig. 3(b)(c). Further, we also provide here the numerical values of the eigenvectors matrix $\mathbf{P}$ (associated with the optically measured, electrically measured and heuristic $\mathbf{T}$ matrix), which were used in the experiment of Fig. 3(a) to define new coordinates $\delta \boldsymbol{\Psi}=$ $\mathbf{P}^{-1} \delta \boldsymbol{\Phi}$.

From the optical measurement of the mutually induced wavelength shift of the MRR resonances, we obtain

$$
\mathbf{T}_{m, \text { opt }}=\left(\begin{array}{cccc}
0 & 0.64 & 0.42 & 0.64 \\
0.44 & 0 & 0.44 & 0.2 \\
0.5 & 0.25 & 0 & 1 \\
0.48 & 0.48 & 0.72 & 0
\end{array}\right)
$$

whose off-diagonal elements [see Fig. 3(b)] are normalized to the max value of 0.173 , and

$$
\mathbf{P}_{\text {opt }}=\left(\begin{array}{cccc}
0.46 & 0.28 & -0.36 & -0.12 \\
0.44 & 0.74 & 0.61 & 0.39 \\
0.51 & -0.23 & 0.44 & 0.54 \\
0.57 & -0.55 & -0.54 & -0.73
\end{array}\right) .
$$

By electrically measuring the variation of heater resistance induced by mutual thermal crosstalk, we obtain

$$
\mathbf{T}_{m, \text { Elec }}=\left(\begin{array}{cccc}
0 & 0.72 & 0.76 & 0.9 \\
0.72 & 0 & 0.63 & 0.83 \\
0.88 & 0.86 & 0 & 1 \\
0.83 & 0.83 & 0.74 & 0
\end{array}\right)
$$

whose off-diagonal elements [see Fig. 3(c)] are normalized to the max value of 0.01 , and

$$
\mathbf{P}_{\text {Elec }}=\left(\begin{array}{cccc}
0.5 & 0.22 & -0.54 & -0.32 \\
0.49 & -0.85 & -0.30 & -0.38 \\
0.45 & 0.47 & 0.78 & -0.16 \\
0.54 & 0.04 & -0.04 & 0.85
\end{array}\right) .
$$

In the heuristic approximation of the $\mathbf{T}$ matrix we considered off-diagonal elements equal to $\mu=0.1$, which is close to the average value of the off-diagonal terms of the optically measured $\mathbf{T}$ matrix (2), we get

$$
\mathbf{P}_{\text {heur }}=\left(\begin{array}{cccc}
0.05 & 0.83 & -0.21 & 0.5 \\
0.75 & -0.25 & 0.34 & 0.5 \\
-0.64 & -0.11 & 0.57 & 0.5 \\
-0.16 & -0.46 & -0.71 & 0.5
\end{array}\right) .
$$

\section{ACKNOWLEDGMENT}

This work was (partially) performed at Polifab, the microand nanofabrication facility of Politecnico di Milano (www.polifab.polimi.it).

\section{REFERENCES}

[1] G. Choo, S. Cai, B. Wang, C. K. Madsen, K. Entesari and S. Palermo, "Automatic Monitor-Based Tuning of Reconfigurable Silicon Photonic APF-Based Pole/Zero Filters," Journal of Lightwave Technology, vol. 36, no. 10, pp. 1899-1911, 2018.

[2] P. Dong, "Silicon Photonic Integrated Circuits for Wavelength-Division Multiplexing Application," Journal of Selected Topics in Quantum Electronics, vol. 22, no. 6, pp. 370-378, 2016.

[3] P. Dumais et al., "Silicon Photonic Switch Subsystem With 900 Monolithically Integrated Calibration Photodiodes and 64-Fiber Package," Journal of Lightwave Technology, vol. 36, no. 2, pp. 233238, 2018.

[4] L. Shen, L. Lu, Z. Guo, L. Zhou and J. Chen, "Silicon optical filters reconfigured from a $16 \times 16$ Benes switch matrix," Optics Express, vol. 27, no. 12, pp. 16945-16957, 2019.

[5] R. Stabile, A. Albores-Mejia, A. Rohit and K. A. Williams, "Integrated optical switch matrices for packet data networks," Microsystems \& Nanoengineering volume, 2016.

[6] T. Alexoudi et al., "Optics in Computing: From Photonic Network-onChip to Chip-to-Chip Interconnects and Disintegrated Architectures," Journal of Lightwave Technology, vol. 37, no. 2, pp. 363-379, 2019.

[7] M. W. AlTaha, H. Jayatilleka, Z. Lu, J. F. Chung, D. Celo, D. Goodwill, E. Bernier, S. Mirabbasi, L. Chrostowski and S. Shekhar, "Monitoring and automatic tuning and stabilization of a $2 \times 2$ MZI 
optical switch for large-scale WDM switch networks," Optics Express, vol. 27, no. 17, pp. 24747-24764, 2019.

[8] H. Jayatilleka, K. Murray, M. Á. Guillén-Torres, M. Caverley, R. Hu, N. A. F. Jaeger, L. Chrostowski and S. Shekhar, "Wavelength tuning and stabilization of microring-based filters using silicon in-resonator photocond," Opt. Express, vol. 23, no. 19, pp. 25084-25097, 2015.

[9] J. C. C. Mak, W. D. Sacher, T. Xue, J. C. Mikkelsen, Z. Yong and J. Poon, "Automatic Resonance Alignment of High-Order Microring Filters," Quantum Electronics, vol. 51, no. 11, pp. 1-11, 2015.

[10] J. C. C. Mak and . J. K. S. Poon, "Multivariable Tuning Control of Photonic Integrated Circuits," Journal of Lightwave Technology, vol. 35, no. 9, pp. 1531-1541, 2017.

[11] S. Grillanda, F. Morichetti, N. Peserico, P. Ciccarella, A. Annoni, M. Carminati and A. Melloni, "Non-Invasive Monitoring of ModeDivision Multiplexed Channels on a Silicon Photonic Chip," Journal of Lightwave Technology, vol. 33, no. 6, pp. 1197-1201, 2015.

[12] H. Jayatilleka, H. Shoman, L. Chrostowski and S. Shekhar, "Photoconductive heaters enable control of large-scale silicon photonic ring resonator circuits," Optica, vol. 6, pp. 84-91, 2019.

[13] Y. Li and A. W. Poon, "Actively stabilized silicon microrings with integrated surface-state-absorption photodetectors using a slopedetection method," Opt. Express, vol. 24, no. 19, pp. 21286-21300, 2016.

[14] F. Testa et al., "Design and Implementation of an Integrated Reconfigurable Silicon Photonics Switch Matrix in IRIS Project," Journal of Selected Topics in Quantum Electronics, vol. 22, no. 6, pp. 155-168, 2016.

[15] M. Milanizadeh, D. Aguiar, A. Melloni and F. Morichetti, "Canceling Thermal Cross-Talk Effects in Photonic Integrated Circuits," Journal of Lightwave Technology, vol. 37, no. 4, pp. 1325-1332, 2019.

[16] H. Jayatilleka, H. Shoman, R. Boeck, N. A. F. Jaeger, L. Chrostowski and S. Shekhar, "Automatic Configuration and Wavelength Locking of Coupled Silicon Ring Resonators," Journal of Lightwave Technology, vol. 36, pp. 210-218, 2018.

[17] K. Padmaraju, D. F. Logan, T. Shiraishi, J. J. Ackert, A. P. Knights and K. Bergman, "Wavelength Locking and Thermally Stabilizing Microring Resonators Using Dithering Signals," Journal of Lightwave Technology, vol. 32, no. 3, pp. 505-512, 2014.

[18] K. Padmaraju, L.-W. Luo, X. Zhu, M. Glick, R. Dutt, M. Lipson and K. Bergman, "Wavelength locking of a WDM silicon microring demultiplexer using dithering signals," in Optical Fiber Conference, an Francisco, CA, USA, 2014.

[19] A. Annoni, E. Guglielmi, M. Carminati, S. Grillanda, P. Ciccarella, G. Ferrari, M. Sorel, M. J. Strain, M. Sampietro, A. Melloni and F. Morichetti, "Automated Routing and Control of Silicon Photonic Switch Fabrics," Journal of Selected Topics in Quantum Electronics, vol. 22, no. 6, pp. 169-176, 2016.

[20] A. Melloni, F. Morichetti, G. Cusmai, R. Costa, A. Breda, C. Canavesi, and M. Martinelli, "Progress in large integration scale circuits in SiON technology," in Transparent Optical Networks, Rome. Italy, 2007.

[21] P. Orlandi, F. Morichetti, M. J. Strain, M. Sorel, P. Bassi and A. Melloni, "Photonic Integrated Filter With Widely Tunable Bandwidth," Journal of Lightwave Technology, vol. 32, no. 5, pp. 897-907, 2014.

[22] D. Aguiar, M. Milanizadeh, E. Guglielmi, F. Zanetto, G. Ferrari, M. Sampietro, F. Morichetti and A. Melloni, "Automatic Tuning of Silicon Photonics Microring Filter Array for Hitless Reconfigurable AddDrop," Journal of Lightwave Technology, 2019.

[23] A. Dupas, P. Layec, D. Verchere, Q. Pham Van and S. Bigo, "Ultra-fast Hitless 100Gbit/s Real-Time Bandwidth Variable Transmitter with SDN optical control," in Optical Fiber Communications Conference and Exposition (OFC), San Diego, CA, 2018.

[24] R. Soref, "Integrated-photonic switching structures," APL Photonics, 2018.

[25] L. Qiao, W. Tang and T. Chu, "32 32 silicon electro-optic switch with built-in monitors and balanced-status units," Scientific Reports, vol. 7, p. 42306, 2017.
[26] L. Zhuang, C. G. H. Roeloffzen, M. Hoekman, K.-J. Boller and A. J. Lowery, "Programmable photonic signal processor chip for radiofrequency applications," Optica, vol. 2, no. 10, pp. 854-859, 2015.

[27] D. Pèrez, I. Gasulla, J. Capmany and R. A. Soref, "Reconfigurable lattice mesh designs for programmable photonic processors," Opt. Express, vol. 24, pp. 12093-12106, 2016.

Maziyar Milanizadeh (1987) received B.Sc in Electronics Engineering from Isfahan University (Iran) in 2011, with specialization in microwave devices. He has 4 years of experience as research assistant in IURRC (Isfahan university) on X-band power amplifiers, target detection, simulation for tracking systems and array antennas. In 2017 he achieved M.Sc in Telecommunications from Politecnico di Milano. During the thesis and after the graduation, he joined the Photonic Devices Group, focusing on algorithms and techniques for calibration and control of photonics integrated circuits, complex system synthesis and modeling, thermal and stress simulations. He is currently attending PhD program at Politecnico di Milano.

Sara Ahamdi She recieved B.Sc from Iran Univ. of Science and technology in Telecommunication-Microwave Engineering and M.Sc from Politecnico di Milano in TelecommunicationTechnology Engineering. She joined photonic device group of Politecnico in 2017 to do the master thesis and after graduation continued there as a PIC design collaborator. Her research interests varies from Integrated Photonics to Microwave photonics, mostly focused on design and control algorithm development.

Matteo Petrini, (1995) received the B.Sc in Computer Science and Engineering (cum laude) in 2017 and the M.Sc in Telecommunication Engineering (cum laude) in 2019, both from Politecnico di Milano. He is currently a Ph.D. Student in the Department of Electronics, Information and Bioengineering of Politecnico di Milano.

$\mathrm{He}$ is focusing on techniques for automatic tuning and testing of Photonic Intregrated Circuits.

Douglas Aguair B.Sc in Electronic Engineering from UFPE, Recife/Brazil, specialized in optical networks, has experience on the development of products for the Telecommunications market, including ASON/GMPLS control plane for OTN networks, ROADMs, DWDM transponders, EDFAs, Submarine systems, and also Metro Ethernet layer 2 equipment. $\mathrm{He}$ has a special interest on the modeling of the nonlinear light propagation in fiber optics, and its applications on high capacity networks. Along the years, has acquired knowledge on topics related to the development process of embedded, high technology, complex systems, specially topics related to requirements gathering, analysis and management. He has skills on complex system modeling and is fascinated on how to combine these high-end products with an agile development process.

Riccardo Mazzanti (1994) graduated from Politecnico di Milano in Telecommunication Engineering in 2019. During M.Sc thesis he collaborated with Photonic Devices Group for 
the design and fabrication of an innovative TOADM filter for new generation optical networks. After graduation he joined PhotonPath where his functions are to innovate and drive original projects in PICs, designing and evaluating architectural trade off of complex photonics integrated networks, including optical modulators, photodetectors, optical amplifiers, multiplexers and control loops. He is also responsible for interfacing with foundries to follow up the fabrication of devices and function at a high level in the lab, testing PICs, writing automation software, running experiments, and debugging electronics.

Francesco Zanetto (1992) received the Bachelor and Master degrees (both summa cum laude) in electronics engineering from Politecnico di Milano in 2014 and 2016 respectively. He's currently a PhD student in the Department of Electronics, Information and Bioengineering of Politecnico di Milano, where he's also been research assistant for six months, and he's working on the development of low-noise analog and mixedsignal electronic instrumentation and measurement systems for silicon photonic applications.

Emanuele Guglielmi (1991) received his Bachelor's degree (cum laude) and Master's degree in Electronic Engineering, and Ph.D. degree (cum laude) in Electronics from Politecnico di Milano, Milano, Italy, in 2013, 2015 and 2019, respectively. In 2018, he spent 6 months at the Massachusetts Institute of Technology (MIT) to work on the subject of photonicelectronic integration.

$\mathrm{He}$ has been working in the field of integrated photonics developing electronic control systems for complex photonics circuits. He is specialized in high-sensitivity measurements and in the design of custom low-noise readout electronics, having experience in developing discrete component boards (PCB) and CMOS integrated circuits.

Marco Sampietro (1957) received his Master Degree in Nuclear Engineering in 1982 at Politecnico di Milano (Italy) and is now full professor of Electronic Circuits and Devices at Politecnico of Milano. He is responsible for the activities in high-sensitivity instrumentation for the nanoscience, with focus on the design of electronic integrated circuits for the measurement of currents, voltages, impedances and noise to access the electronic properties of nano-bio sensors. He has been the coordinator of many national and international research projects and scientific partner in six large-size European projects. He is co-author of more than 200 peerreviewed publications and holds 5 patents. From 2012 to 2018 ha has been the Dean of the Bachelor I\& Master Course Program in "Electronics Engineering" at Politecnico di Milano.

Francesco Morichetti Assistant Professor at Politecnico di Milano, received a master's degree (cum laude) in Telecommunication Engineering in 2001 and a Ph.D. degree (cum laude) in Information Engineering in 2008 from Politecnico di Milano. In 2001, he joined the Integrated Optics Lab of CoreCom, Italy. In 2009, he was appointed head of the Integrated Optics Lab at Politecnico di Milano. From 2012 to
2017, he was a postdoctoral researcher at Politecnico di Milano. His research interests include the modeling, design, and characterization of photonic integrated circuits for optical signal processing. $\mathrm{He}$ is the coauthor of three chapters of international books, more than 200 papers in international journals and conference proceedings, and five international patents. He is a member of the scientific panel of Spotlight on Optics (Optical Society of America).

Andrea Melloni OSA Fellow, Full Professor at Politecnico di Milano-Italy in Electromagnetic fields, leader of the Photonic Devices group. His field of research is in the analysis, design, characterization and exploitation of integrated optical devices for optical communication and sensing. He has been one of the pioneers of the slow light concept; contributed to define the new schemes of generic photonic foundries in Europe; is now focusing on the control and stabilization of photonic integrated circuits. He is co-inventor of the first noninvasive on-chip lightmonitor. In 2008 he founded the company Filarete, for the development Aspic, the first circuit simulator for integrated optical circuits. He has been one of the proponents, and now deputy director, of Polifab, the facility for micro and nano technologies at Politecnico di Milano. 